\title{
SPHERES OF SMALL DIAMETER WITH LONG SWEEP-OUTS
}

\author{
YEVGENY LIOKUMOVICH
}

(Communicated by Lei $\mathrm{Ni}$ )

\begin{abstract}
We prove the absence of a universal diameter bound on lengths of curves in a sweep-out of a Riemannian 2-sphere. If such a bound existed it would yield a simple proof of the existence of short geodesic segments and closed geodesics on a sphere of small diameter.
\end{abstract}

\section{INTRODUCTION}

By a sweep-out of a Riemannian 2-sphere $M=\left(S^{2}, g\right)$ we mean a noncontractible loop $\gamma_{t}$ in $\left(\Lambda M, \Lambda^{0} M\right)$, where $\Lambda M$ denotes the space of free loops on $M$ and $\Lambda^{0} M$ denotes the space of constant loops. In other words, $\gamma_{t}$ is a 1-parameter family of closed curves starting and ending at a point and inducing a nonzero degree map $f: S^{2} \rightarrow M$. If there exists a sweep-out by loops of length $\leq L$, then the standard minimax argument implies that there exists a nontrivial closed geodesic on $M$ of length $\leq L[\mathrm{~B}$.

Moreover, A. Nabutovsky and R. Rotman demonstrate in NR1 that existence of a certain sweep-out, called an $L$-slicing, of the sphere by loops of length $\leq 2 L$ implies that for any two points $a, b \in M$ there exist $k$ distinct geodesic segments from $a$ to $b$ of length $\leq 2 k L+2 \operatorname{diam}(M)$. If we could take $L \leq C \operatorname{diam}(M)$ we would obtain a linear in $k$ bound on the length of the $k$-th shortest geodesic segment in terms of the diameter.

Yet A. Nabutovsky and R. Rotman observe (see [N], [NR1, [NR2]) that an example of S. Frankel and M. Katz [FK] (see below) suggests that for any $C$ there exists a sphere with no sweep-out obeying this inequality. The author learned from Regina Rotman that a similar conjecture was independently made by S. Sabourau. In this paper we prove this conjecture.

\section{MAin RESUlt}

Let $\gamma: I \times S^{1} \rightarrow M$ be a 1-parameter family of free loops (we write $\gamma_{t}(s)$ for $\gamma(t, s))$, such that $\gamma_{0}(s)$ and $\gamma_{1}(s)$ are constant loops. $\gamma$ induces a map $f: S^{2} \rightarrow M$, such that $\gamma(t, s)=f \circ p(t, s)$, where $p: I \times S^{1} \rightarrow S^{2}$ is the suspension map that collapses $\{0\} \times S^{1}$ to the south pole and $\{1\} \times S^{1}$ to the north pole of $S^{2}$. If $\operatorname{deg}(f) \neq 0$ we call $\gamma_{t}$ a sweep-out.

Theorem 1. For any $C>0$ there exists a Riemannian 2-sphere $M$ of diameter $\leq 1$, such that for any sweep-out $\gamma_{t}$ of $M$ there is a loop $\gamma_{t_{0}}$ of length $\geq C$.

Received by the editors June 2, 2011.

2010 Mathematics Subject Classification. Primary 53C23.

The author's research is supported by an NSERC CGS scholarship. 
F. Balacheff and S. Sabourau in [BS] defined a diastole of $M$ as

$$
\operatorname{dias}(M):=i n f_{\left(\gamma_{t}\right)} \sup _{0 \leq t \leq 1} \text { length }\left(\gamma_{t}\right),
$$

where $\left(\gamma_{t}\right)$ runs over the families of loops inducing $f: S^{2} \rightarrow M$ of degree \pm 1 . In $\underline{S}$, Remark 4.10] S. Sabourau constructs a sequence $M_{n}$ of Riemannian two-spheres such that

$$
\lim _{n \rightarrow \infty} \frac{\sqrt{\operatorname{Area}\left(M_{n}\right)}}{\operatorname{dias}\left(M_{n}\right)}=0 .
$$

Theorem 1 implies an analogous result with $\operatorname{diam}\left(M_{n}\right)$ in place of $\sqrt{\operatorname{Area}\left(M_{n}\right)}$.

F. Balacheff and S. Sabourau prove [BS] that if 1-parameter families of loops in the definition of the diastole are replaced with 1-parameter families of one-cycles, then every Riemannian 2-sphere satisfies $\operatorname{dias}(M) \leq C \sqrt{\operatorname{Area}(M)}$ for a universal constant $C$.

Proof. We use an example of S. Frankel and M. Katz [FK]. For any natural number $N$ they embed a binary tree $T$ of height $N$ in a 2-dimensional disc $D$ and define a Riemannian metric on $D$, such that the distance between any two nonadjacent edges of $T$ is $\operatorname{dist}\left(e_{i}, e_{j}\right) \geq 1 / 2$, but the diameter $\operatorname{diam}(D) \leq 1$. They prove that for every homotopy of closed curves $\gamma_{t}$ with $\gamma_{0}=\partial D$ and $\gamma_{1}=\{*\}$ there is an intermediate curve $\gamma_{t_{0}}$ that intersects $O(N / \log N)$ edges of $T$ (i.e. $\geq C^{\prime} \frac{N}{\log N}$ for some universal constant $C^{\prime}>0$ and all big enough $\left.N\right)$. They conclude that $\gamma_{t_{0}}$ has length $O(N / \log N)$.

Let $M=\left(S^{2}, g\right)$ be a sphere of diameter less than 1 containing a disc of Frankel and Katz with an embedded binary tree $T$ of height $N$. Consider a sweep-out $\gamma_{t}$ and let $f: S^{2} \rightarrow M$ be the induced map from the suspension of $S^{1}$ to $M$ $\left(\gamma_{t}(s)=f \circ p(t, s)\right)$.

Suppose that at some time $t_{0} \in[0,1], \gamma_{t_{0}}$ does not pass through a vertex $v \in T$. Let $E$ denote a connected component of $M \backslash \gamma_{t_{0}}$ that contains $v$. Identify all points of $M \backslash E$ to a new point $x$ to obtain a quotient space $E \cup\{x\}$ homeomorphic to $S^{2}$. The loop $p\left(t_{0}, s\right)$ divides $S^{2}$ into two connected components, the south component $S_{t_{0}}=\left\{p(t, s): t<t_{0}\right\}$ and the north component $N_{t_{0}}=\left\{p(t, s): t>t_{0}\right\}$. A composition of $f$ with the quotient map $q_{1}: M \rightarrow E \cup\{x\}$ maps the loop $p\left(t_{0}, s\right)$ to the point $x$. If we collapse all points of $p\left(t_{0}, s\right)$ to a point $y$, we obtain a map $f_{t_{0}, v}: S_{t_{0}} \cup\{y\} \cup N_{t_{0}} \rightarrow E \cup\{q\}$, where the quotient space $S_{t_{0}} \cup\{y\} \cup N_{t_{0}}$ is homeomorphic to the wedge sum $S^{2} \vee S^{2}$. We have the following commutative diagram:

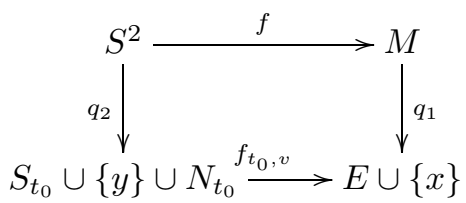

Let $f_{t_{0}, v}^{S}$ denote the restriction of $f_{t_{0}, v}$ to the south sphere $S_{t_{0}} \cup\{y\}$ and $f_{t_{0}, v}^{N}$ denote the restriction to the north sphere $N_{t_{0}} \cup\{y\}$. Observe that from the induced commutative diagram for the second homology groups we have $\operatorname{deg}(f)=$ $\operatorname{deg}\left(f_{t_{0}, v}^{S}\right)+\operatorname{deg}\left(f_{t_{0}, v}^{N}\right)$. Indeed, the map $q_{2 \sharp}: H_{2}\left(S^{2}\right) \rightarrow H_{2}\left(S^{2} \vee S^{2}\right)$ sends a generator 1 to an element $(1,1) \in \mathbb{Z} \times \mathbb{Z}$ and $\left(f_{t_{0}, v}\right)_{\sharp}(a, b)=\left(f_{t_{0}, v}^{S}\right)_{\sharp}(a)+\left(f_{t_{0}, v}^{N}\right)_{\sharp}(b)$, while $q_{1 \sharp}$ is an isomorphism. 
Let $A \subset[0,1]$ denote the set of all $t$ such that $\gamma_{t}$ does not pass through $v$. We define a function $d_{v}: A \rightarrow \mathbb{N}$ (degree of $v$ at time $t$ ) by

$$
d_{v}(t)=\operatorname{deg}\left(f_{t, v}^{N}\right) .
$$

We need two simple facts about $d_{v}(t)$.

Observation 1. If $\gamma_{t}$ does not pass through $v$ for $t \in\left[t_{1}, t_{2}\right]$, then $d_{v}(t)$ is constant on $\left[t_{1}, t_{2}\right]$.

Proof. Choose a small disc $D$ around $v$ such that $D \cap f\left(p\left(\left[t_{1}, t_{2}\right]\right)\right.$ is empty and define a quotient map $q$ collapsing $M \backslash D$ to a point. For each $t \in\left[t_{1}, t_{2}\right]$ we can define a map between spheres $q^{\prime}: N_{t} \cup\{a\} \rightarrow N_{t_{2}} \cup\{b\}$ sending $N_{t} \backslash N_{t_{2}}$ to $b$. Then $q \circ f_{t, v}^{N}=q \circ f_{t_{2}, v}^{N} \circ q^{\prime}$. As $\operatorname{deg}(q)=\operatorname{deg}\left(q^{\prime}\right)=1$ we have $\operatorname{deg}\left(f_{t, v}^{N}\right)=\operatorname{deg}\left(f_{t_{2}, v}^{N}\right)$.

Observation 2. Let $v_{1}, v_{2}$ be two vertices of $T$ connected by an edge $e$. If $\gamma_{t}$ does not intersect $e$, then $d_{v_{1}}(t)=d_{v_{2}}(t)$.

Proof. Since $\gamma_{t}$ does not intersect $e, v_{1}$ and $v_{2}$ belong to the same connected component of $M \backslash \gamma_{t}$. Hence, $f_{t, v_{1}}^{N}=f_{t, v_{2}}^{N}$.

Without any loss of generality we may assume that the images of north and south poles under $f$ are not vertices of $T$. We observe that $d_{v}(0)=\operatorname{deg}(f) \neq 0$ and $d_{v}(1)=0$ for all vertices $v$.

The rest of the proof proceeds as in FK]. Let $V$ be the set of vertices of $T$ and $K(t)=\#\left\{v \in V: d_{v}(t)=0\right\}$. We may perturb the homotopy slightly, so that $\gamma_{t}$ passes through no more than one vertex for each $t$. Observation 1 implies that as $t$ varies between 0 and $1, K(t)$ will attain every value between 0 and $2^{N}-1$ (recall that $N$ is the height of $T$ ).

Consider what values $K(t)$ can attain if $\gamma_{t}$ intersects only one edge of $T$. Let $v_{1}$ and $v_{2}$ be two vertices of this edge $e$ at distances (in the standard metric on the tree) $i$ and $i+1$, respectively, from the root. By Observation 2 we have the following possibilities for the value of $K(t)$ : the number of vertices in the connected component of $M \backslash \gamma_{t}$ that contains $v_{1}\left(2^{N}-2^{N-i-1}\right)$, the number of vertices in the connected component that contains $v_{2}\left(2^{N-i-1}-1\right)$ or one of the min or max values $\left(0\right.$ and $\left.2^{N}-1\right)$. Since this is true for every distance from the root $i, K(t)$ can attain at most $2(N-1)+2$ different values if $\gamma_{t}$ intersects only one edge of $T$. Suppose there are exactly $j$ intersections. Then the number of possible different values $K(t)$ can attain is smaller than or equal to the product of the numbers of different values it can attain for each intersection, i.e. $(2 N)^{j}$. If throughout the homotopy $\gamma_{t}$ intersects at most $k$ edges, then $K(t)$ attains no more than $\sum_{j=1}^{k}(2 N)^{j} \leq(2 N)^{k+1}$ distinct values. Since all possible values are attained, we have $(2 N)^{k+1} \geq 2^{N}-1$, and hence $k \geq O(N / \log N)$.

Since the distance between any two nonadjacent edges is greater than $1 / 2$, we have that for some $t, \gamma_{t}$ will be at least $O(N / \log N)$ long.

\section{ACKNOWLEDGEMENTS}

The author gratefully acknowledges the support by a Natural Sciences and Engineering Research Council (NSERC) CGS scholarship. The author would like to thank Professors Alexander Nabutovsky and Regina Rotman for explaining the question to him and for many very helpful discussions. The author is thankful to an anonymous referee whose comments helped to improve the exposition. 


\section{REFERENCES}

[B] G. D. Birkhoff, Dynamical systems, Amer. Math. Soc. Colloq. Publ., Vol. 9, Providence, RI, 1966. MR0209095 (35:1)

[BS] F. Balacheff, S. Sabourau, Diastolic and isoperimetric inequalities on surfaces, Ann. Sci. École Norm. Sup. 43 (2010), 579-605. MR2722509

[FK] S. Frankel, M. Katz, The Morse landscape of a Riemannian disc, Annales de l'Inst. Fourier 43 (1993), no. 2, 503-507. MR.1220281 (94c:53057)

[N] A. Nabutovsky, Effective universal coverings and local minima of the length functional on loop spaces, Geom. Funct. Anal. 20 (2010), no. 2, 545-570. MR2671286

[NR1] A. Nabutovsky, R. Rotman, Length of geodesics on a two-dimensional sphere, American J. Math. 131 (2009), 545-569. MR2503992 (2010g:53071)

[NR2] A. Nabutovsky, R. Rotman, Short geodesic segments between two points on a closed Riemannian manifold, Geom. Funct. Anal. 19 (2009), no. 2, 498-519. MR2545247 (2011b:53086)

[S] S. Sabourau, Filling radius and short closed geodesics of the 2-sphere, Bull. Soc. Math. France 132 (2004), 105-136. MR2075918(2005g:53065)

Department of Mathematics, University of Toronto, Toronto, Ontario M5S 2E4, CANADA

E-mail address: e.liokumovich@utoronto.ca 